\title{
Excessive social media users demonstrate impaired decision making in the Iowa Gambling Task
}

\author{
DAR MESHI $^{1 *}$, ANASTASSIA ELIZAROVA ${ }^{2}$, ANDREW BENDER $^{3,4}$ and ANTONIO VERDEJO-GARCIA ${ }^{5}$ \\ ${ }^{1}$ Department of Advertising and Public Relations, Michigan State University, East Lansing, MI, USA \\ ${ }^{2}$ Department of Neurology and Neurosurgery, Montreal Neurological Institute, McGill University, Montreal, Canada \\ ${ }^{3}$ Department of Epidemiology and Biostatistics, Michigan State University, East Lansing, MI, USA \\ ${ }^{4}$ Department of Neurology and Ophthalmology, Michigan State University, East Lansing, MI, USA \\ ${ }^{5}$ School of Psychological Sciences, Monash Institute of Cognitive and Clinical Neurosciences, Monash University, \\ Melbourne, VIC, Australia
}

(Received: July 2, 2018; revised manuscript received: October 22, 2018; second revised manuscript received: November 26, 2018; accepted: December 2, 2018)

\begin{abstract}
Background and aims: Online social networking sites (SNSs) like Facebook provide users with myriad social rewards. These social rewards bring users back to SNSs repeatedly, with some users displaying maladaptive, excessive SNS use. Symptoms of this excessive SNS use are similar to symptoms of substance use and behavioral addictive disorders. Importantly, individuals with substance use and behavioral addictive disorders have difficulty making value-based decisions, as demonstrated with paradigms like the Iowa Gambling Task (IGT); however, it is currently unknown if excessive SNS users display the same decision-making deficits. Therefore, in this study, we aimed to investigate the relationship between excessive SNS use and IGT performance. Methods: We administered the Bergen Facebook Addiction Scale (BFAS) to 71 participants to assess their maladaptive use of the Facebook SNS. We next had them perform 100 trials of the IGT to assess their value-based decision making. Results: We found a negative correlation between BFAS score and performance in the IGT across participants, specifically over the last block of 20 trials. There were no correlations between BFAS score and IGT performance in earlier blocks of trials. Discussion: Our results demonstrate that more severe, excessive SNS use is associated with more deficient valuebased decision making. In particular, our results indicate that excessive SNS users may make more risky decisions during the IGT task. Conclusion: This result further supports a parallel between individuals with problematic, excessive SNS use, and individuals with substance use and behavioral addictive disorders.
\end{abstract}

Keywords: social media, Facebook, addiction, decision making, Iowa Gambling Task, risk

\section{INTRODUCTION}

Two and a half billion people around the world regularly use social networking sites (SNSs), such as Facebook, Instagram, and Twitter, and this number increases every day as more people gain access to the Internet (Statista, 2018). SNS users are not just creating profiles, they are spending substantial, recurring periods of time on these platforms observing and interacting with others. For example, in 2016, Facebook reported that the average user spends 50 min a day on its sites (Stewart, 2016). This ubiquitous and timeconsuming use results from the reinforcing effects of social rewards, as SNSs provide frequent and copious social rewards to their users (Meshi, Tamir, \& Heekeren, 2015).

In some individuals, this behavioral reinforcement on SNSs may lead to maladaptive, excessive SNS use (Griffiths, Kuss, \& Demetrovics, 2014). Excessive SNS users display a preoccupation with SNS platforms when they are not using them, mood modification when they access these sites, and tolerance to the social rewards obtained on these sites. These excessive SNS users also experience conflict with others because of their use, and when attempting to quit, they display withdrawal symptoms and often relapse. Case studies also report excessive SNS users seeking clinical evaluation and treatment (Griffiths et al., 2014; Karaiskos, Tzavellas, Balta, \& Paparrigopoulos, 2010). The severity of this excessive SNS use and its potential to be termed as an "addiction" are hotly debated (Carbonell \& Panova, 2017), and importantly, excessive SNS use is not currently included in the fifth edition of Diagnostic and Statistical Manual of Mental Disorders (DSM-5; American Psychological Association, 2013). Nevertheless, there exists a clear parallel between the symptoms of substance use and behavioral addictive disorders, as defined by the DSM-5, and the symptoms of excessive SNS use.

* Corresponding author: Dar Meshi, PhD; Department of Advertising and Public Relations, Michigan State University, 404 Wilson Road, East Lansing, MI 48824, USA; Phone: +1 517355 1282; Fax: +1 517432 2589; E-mail: darmeshi@gmail.com

This is an open-access article distributed under the terms of the Creative Commons Attribution-NonCommercial 4.0 International License, which permits unrestricted use, distribution, and reproduction in any medium for non-commercial purposes, provided the original author and source are credited, a link to the CC License is provided, and changes - if any - are indicated. 
Indeed, the parallel between excessive SNS use and substance use disorder does not stop at behavior; recent neuroimaging work has also demonstrated similarities. For example, the brain's reward system - which is composed of regions such as the striatum and amygdala - responds to drug consumption (relevant to substance use disorder), monetary rewards (relevant to gambling disorder), and social rewards (relevant to excessive SNS use; Meshi, Morawetz, \& Heekeren, 2013; Suckling \& Nestor, 2017). Furthermore, morphological differences in these brain structures overlap; for example, the striatum and amygdalae are smaller in both excessive SNS users and substance abusers (He, Turel, Brevers, \& Bechara, 2017; Suckling \& Nestor, 2017).

These brain regions compute value during decision making (Bartra, McGuire, \& Kable, 2013), and as one would expect, individuals with substance use and behavioral addictive disorders have difficulty making value-based decisions (Bechara \& Martin, 2004). Researchers have designed laboratory paradigms, such as the Iowa Gambling Task (IGT), to reveal this impaired decision making (Bechara, Damasio, Damasio, \& Anderson, 1994). Individuals dependent on various substances, such as opioids, cocaine, methamphetamines, marijuana, alcohol, and nicotine, demonstrate deficient performance in the IGT, as well as individuals with gambling disorder (for review, see Buelow \& Suhr, 2009). Importantly, however, with regard to excessive SNS users, it is currently unknown if these individuals display the same difficulty making decisions as individuals with substance use and behavioral addictive disorders. With the above similarities in mind, we hypothesized that individuals who display excessive SNS use will also display impaired value-based decision making. To address our hypothesis, we examined individual differences with respect to excessive Facebook use as assessed by the Bergen Facebook Addiction Scale (BFAS; Andreassen, Torsheim, Brunborg, \& Pallesen, 2012) and performance in the IGT. We focused on the Facebook platform, because it is currently the most widely used SNS around the world, with 1.47 billion daily active users as of June 2018 (Facebook, 2018). In addition, we assessed levels of depression in our sample to control for it in our analysis depressive symptoms have been shown to correlate with degree of SNS use (for review, see Baker \& Algorta, 2016), and depression has also been associated with impaired performance in the IGT (Must et al., 2006).

\section{METHODS}

\section{Participants}

Seventy-one participants (44 females) between 18 and 35 years of age $[($ mean $=23.7$, standard deviation $(S D)=3.8$ years] took part in this study, which was conducted at a large German university. Individuals were recruited through posted flyers. To note, all participants were proficient in the English language, and English was used for the recruitment flyers, e-mail correspondence, face-to-face interactions, and the below-described experimental measures. All participants self-reported having no history of psychiatric disorders, including substance use disorder or other behavioral disorders (e.g., gambling disorder). After completion of the experiment, all individuals were provided $10 €$ for their participation.

\section{Measures}

We collected measures of excessive SNS use, value-based decision making, and depression:

Excessive Facebook use. We used the BFAS (Andreassen et al., 2012) to assess excessive Facebook use. The BFAS consists of six items rated on a 5-point Likert scale $(1=$ very rarely; $5=$ very often $)$. Therefore, when participants' responses are summed, final scores can range from 6 to 30 points. Each BFAS item assesses a commonly accepted core aspect of addiction: salience (preoccupation), mood modification, tolerance, conflict, withdrawal, and relapse (Griffiths et al., 2014). Reliability and validity of the BFAS have been established (Andreassen et al., 2012), and the internal consistency with our sample was good (Cronbach's $\alpha=.87$ ). BFAS scores were normally distributed and no participant's score was more than three $S D$ s from the mean.

Decision making. All participants performed 100 trials of a computerized version of the IGT (Dancy \& Ritter, 2017) to assess value-based decision making (Bechara et al., 1994). In this task, participants see four decks of cards displayed on the screen in front of them (A, B, C, and D). On each trial, participants choose a card and receive a specified amount of play money reward for this choice. Interspersed among these rewards are punishments, consisting of play money losses of different amounts. Importantly, two of the decks (A and B) produce high immediate gains $(\$ 100)$ for each choice, but they also provide sizeable punishments - in the long run, these decks take more money than they give. We term these decks, disadvantageous. Two other decks ( $\mathrm{C}$ and D) produce low immediate gains (\$50) for each choice, and they also provide smaller punishments compared to the other decks - in the long run, these decks give more money than they take. We term these decks, advantageous.

Before performing the task, participants are told that the goal is to make as much money as possible and to avoid losing as much money as possible. They are also told that they can pick cards from any deck, and switch whenever they want. Participants are also informed that some decks are better than others and that if they want to do well, they should avoid the bad decks and choose cards from the good decks.

To note, different versions of the IGT have been used in the literature, with some researchers providing the above hint to stay away from the bad decks and other researchers not providing the hint. Furthermore, some researchers have used play money, whereas others have used real money. We chose to provide the hint and to use play money, as previous research has demonstrated more advantageous card choices in healthy individuals with these conditions (Fernie \& Tunney, 2006). As we hypothesized impaired decision making in the IGT by excessive SNS users, this experimental set up would yield the highest likelihood of finding our hypothesized effect.

For analysis, we divided the 100-trial experiment into five blocks of 20 trials each and calculated scores for the task (IGT score) in each block by subtracting the total 
number of cards chosen from the disadvantageous decks (A and B), from the total number of cards chosen from the advantageous decks (C and D). Higher IGT Scores indicate that the participant performed better on the task. Total IGT scores (mean $=15.1, S D=19.0)$ were normally distributed and no participant's score was more than three $S D$ s from the mean.

Depression. We also assessed participants' level of depression with the Beck Depression Inventory II (BDI; Beck, Steer, Ball, \& Ranieri, 1996). The BDI is one of the most commonly used instruments to assess depression and consists of 21 items rated on a 4-point Likert scale. We administered the BDI because several previous studies reported a positive correlation between depressive symptoms and online social networking, although several other studies have either failed to replicate this finding, or revealed a negative correlation (for review, see Baker \& Algorta, 2016). Nevertheless, we assessed depressive symptoms in our sample with the BDI to control for it in our analyses. The reliability of the BDI with our sample was good (Cronbach's $\alpha=.86$ ); however, BDI data were not normally distributed. They were positively skewed (skewness $=1.64, S E=0.29$ ) and displayed kurtosis (kurtosis = $4.73, S E=0.56$ ), with two participants more than three $S D \mathrm{~s}$ away from the mean $($ mean $=12.4$, median $=12.0, S D=$ 8.8). To address this, we winsorized the two highest and lowest values to equal the third highest and lowest value, respectively (94\% winsorization). This resulted in normally distributed data with no outliers $($ mean $=11.89$, median $=$ $12.00, S D=7.11$, skewness $=0.43, S E=0.29$, kurtosis $=$ $-0.47, S E=0.56$ ). This winsorized data set was used for all reported BDI analyses.

\section{Procedure}

After responding to our recruitment flyer, participants were sent an electronic survey to assess their age, gender, and BFAS score. Participants were then invited into our behavioral testing lab. In a closed room with no distractions, participants were provided instructions for the IGT and then performed the task on a computer. After completion of the task, participants filled out the BDI, and were then paid and debriefed.

\section{Statistical analyses}

All analyses were performed using SPSS software (IBM Inc., version 25, Armonk, NY, USA). First, as a manipulation check, we conducted a repeated-measures analysis of variance (ANOVA) with block IGT scores to determine if participants learned to choose the advantageous decks during the 100-trial experiment. Next, we addressed our hypothesized negative correlation between excessive use of Facebook, as measured by BFAS score and performance on the IGT. To do this, we conducted one-tailed, first-order partial correlations between BFAS scores and IGT scores for all 20-trial blocks, while controlling for depression (BDI). We also conducted two-tailed, zero-order correlation analyses between all collected survey measures: BFAS, BDI, age, and gender. All correlations were conducted with continuous variables, except correlations with gender (which is a dichotomous variable), so these correlations were point biserial. All reported significant results survived Bonferroni correction for multiple comparisons.

\section{Ethics}

Study procedures were carried out in accordance with the Declaration of Helsinki and approved by the ethics review committee of a large European university. All participants were informed about the study and all provided informed consent for participation.

\section{RESULTS}

Participants displayed a wide range of BFAS scores (mean = $15.1, S D=5.8$, range $=6-27)$. BFAS scores did not correlate with age $(r=-.13, p=.14,95 \% \mathrm{CI}=-0.17 / 0.29)$ or gender $(r=-.02, p=.43,95 \% \mathrm{CI}=-0.28 / 0.22)$, and age and gender were not correlated with each other $(r=-.06$, $p>.05,95 \% \mathrm{CI}=-0.33 / 0.19)$. BFAS scores did correlate with BDI scores $(r=.43, p<.001,95 \% \mathrm{CI}=0.21 / 0.63)$, which is congruent with some previous research, as mentioned in the "Methods" section. Furthermore, age $(r=-.13$, $p>.05,95 \% \mathrm{CI}=-0.32 / 0.07)$ and gender $(r=.06, p>.05$, $95 \% \mathrm{CI}=0.10 / 0.25)$ were not correlated with BDI.

Group performance across the 100-trial IGT, organized into 20-trial blocks, is depicted in Figure 1 (block 1: mean = $-3.7, S D=3.4$; block 2: mean $=1.5, S D=5.9$; block 3: mean $=4.5, S D=7.4$; block 4 : mean $=6.1, S D=7.6$; block $5:$ mean $=6.7, S D=7.8$ ). Repeated-measures ANOVA with block IGT scores revealed that as the experiment progressed, participants choose more advantageous decks than disadvantageous decks $\left[F_{(4,280)}=31.9, p<.01\right.$, partial $\left.\eta^{2}=0.31\right]$. Post-hoc paired $t$-tests revealed that mean IGT scores for blocks 1 and 2 were significantly different from all other blocks (for all comparisons: $t$ 's $>3.0, p$ 's $<.01$ ), however, mean IGT scores for blocks 3-5 were not statistically different from each other $(p$ 's $>.05)$. Our significant repeated measures finding with a large effect size demonstrates that, as a group, participants were performing the task as instructed and able to learn from the rewards and punishments they received as a result of their deck choices.

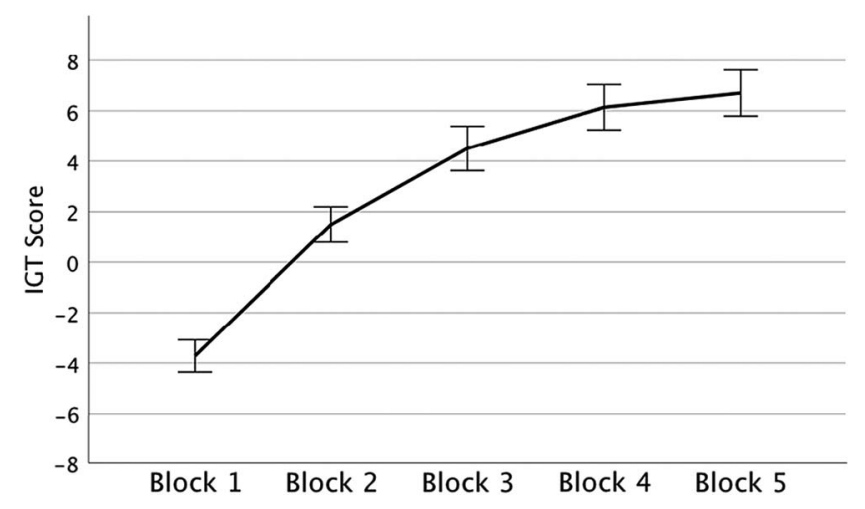

Figure 1. IGT scores [(Decks C + D) $-($ Decks A + B) ] for each block of 20 trials across the 100-trial experiment. As the experiment progressed, participants choose more advantageous decks than disadvantageous decks. Error bars $= \pm 1$ SEM 


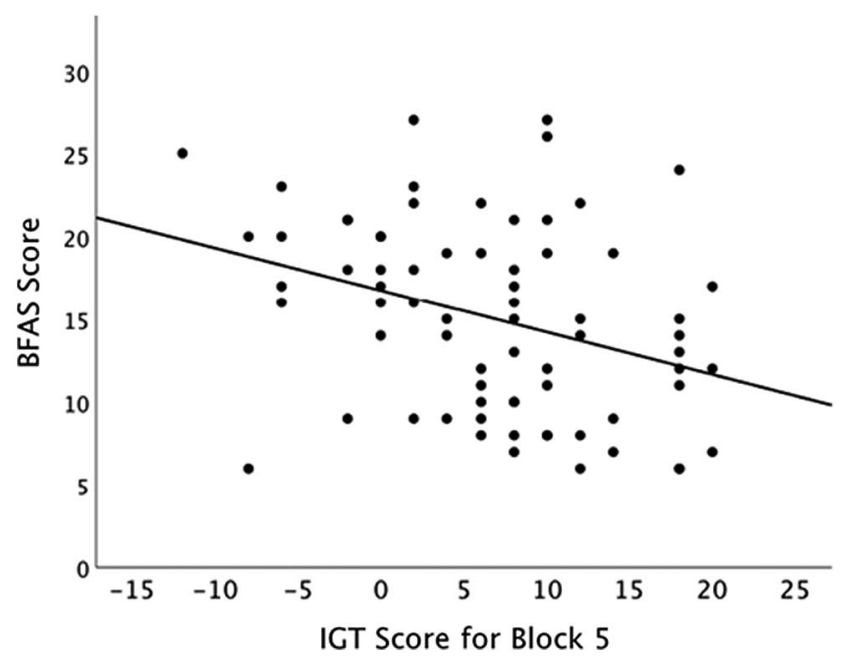

Figure 2. IGT score for block 5 of the task negatively correlates with BFAS score across participants

We analyzed the relationship between excessive social media use and IGT performance. This revealed a significant negative, first-order partial correlation between BFAS and IGT score for block 5 (trials 81-100), when controlling for BDI $(r=-.31, p<.01,95 \% \mathrm{CI}=-0.52 /-0.07$, one-tailed; Figure 2). A post-hoc power analysis revealed power of 0.85 , demonstrating that this study was well-powered to detect the reported medium effect size. The partial correlation between BFAS and IGT score was not significant in any other block: block 1 (trials $1-20 ; r=-.01, p>.05,95 \%$ $\mathrm{CI}=-0.24 / 0.25$ ), block 2 (trials $21-40 ; r=.23, p>.05$, $95 \% \mathrm{CI}=-0.04 / 0.48$ ), block 3 (trials $41-60 ; r=.01$, $p>.05,95 \% \mathrm{CI}=-0.26 / 0.24$ ), or block 4 (trials $61-80$; $r=.03, p>.05,95 \% \mathrm{CI}=-0.22 / 0.31)$.

To note, there were no significant correlations between BDI and IGT scores across blocks (block $1: r=.21, p>.05$, 95\% CI $=-0.13 / 0.40$; block $2: r=.09, p>.05,95 \% \mathrm{CI}=$ $-0.12 / 0.30$; block 3: $r=-.01, p>.05,95 \% \mathrm{CI}=-0.17 /$ 0.19 ; block $4: r=.07, p>.05,95 \% \mathrm{CI}=-0.13 / 0.25$; block 5: $r=-.15, p>.05,95 \% \mathrm{CI}=-0.33 / 0.05)$.

\section{DISCUSSION}

We observed that more severe, excessive SNS use is associated with worse performance in the last 20 trials of the IGT. Previous research with the IGT has led to investigators categorizing the first 40 trials of the task (blocks 1-2) as decision making under ambiguity, and the last 60 trials (blocks 3-5) as decision making under risk (Bechara, Damasio, Tranel, \& Damasio, 1997; He et al., 2010). Therefore, our results in block 5 imply that excessive SNS users may have difficulty specifically with decision making under risk. To note, however, these previous studies analyzed the first 40 trials as one block and the next 60 trials as another block, and we did not find significant results when we analyzed our data in this way - we found a significant relationship only in the last block of 20 trials (trials 81-100). Future research with different measures can more directly address this implication about excessive SNS use and risky decision making, teasing apart aspects of decision making under both risk and ambiguity.

Our results are consistent with a previous finding demonstrating that BFAS score correlates with another aspect of cognitive impulsivity, delay discounting (Delaney, Stein, \& Gruber, 2017). Research has demonstrated that the delay discounting task and the IGT assess different aspects of impulsive choice (Stevens et al., 2014). Therefore, our results expand on this previous finding, tapping into another facet of decision making, as the IGT taxes decisions based on rewards and punishments - our findings imply that excessive SNS users are weighting the potential positive outcomes of their decisions more than the potential negative outcomes.

This study has limitations that are worth mentioning. First, we only assessed excessive Facebook use and not excessive use across all SNSs. For example, it could be that some participants scored low on excessive Facebook use with the BFAS, but they excessively use another SNS and also display aberrant decision making. Second, the BFAS is a self-report measure, and we did not track participants' actual SNS use to confirm their survey responses. Next, although myriad research has been performed with the IGT and performance has been causally linked to frontal lobe brain structures, the task is not without its critics (Buelow \& Suhr, 2009). Some feel it can be interpreted in several ways (i.e., assessing impulsivity, not risky decision making) and recent reports have questioned its reliability and validity (Schmitz, Kunina-Habenicht, Hildebrandt, Oberauer, \& Wilhelm, 2018). Nevertheless, we demonstrate differences in IGT performance across individuals with respect to excessive Facebook use. Finally, we did not use a clinical sample to compare with healthy controls, rather we looked for a correlation across individuals who displayed a wide range of BFAS scores. Future research can address this limitation by assessing IGT performance of individuals receiving clinical treatment for excessive SNS use.

Our results have important societal implications. SNS use is ubiquitous and continues to grow, likely resulting in more individuals displaying excessive, problematic SNS use. Meanwhile, companies continue to develop features on SNS platforms to make them even more compelling. Taking this into consideration, our current finding, which demonstrates a behavioral similarity between excessive SNS use and substance use and behavioral addictive disorders, can influence the beliefs and practices of policy makers, therapists, and tech industry leaders. Our research provides a reference point for these individuals to discuss and address excessive SNS use in their respective professions.

Funding sources: This study was supported by German Research Foundation (DFG), grant: HE 3347/6-1.

Authors' contribution: DM: study concept and design, statistical analysis, interpretation of data, and obtained funding. AE: collected data and statistical analysis. AB: statistical analysis. AV-G: study supervision and interpretation of data. 
Conflict of interest: The authors report no financial or other relationship relevant to the subject of this article.

\section{REFERENCES}

American Psychological Association. (2013). Diagnostic and statistical manual of mental disorders: DSM-V (5th ed.). Washington, DC: American Psychological Association.

Andreassen, C. S., Torsheim, T., Brunborg, G. S., \& Pallesen, S. (2012). Development of a Facebook Addiction Scale. Psychological Reports, 110(2), 501-517. doi:10.2466/02.09.18. PR0.110.2.501-517

Baker, D. A., \& Algorta, G. P. (2016). The relationship between online social networking and depression: A systematic review of quantitative studies. Cyberpsychology, Behavior, and Social Networking, 19(11), 638-648. doi:10.1089/cyber.2016.0206

Bartra, O., McGuire, J. T., \& Kable, J. W. (2013). The valuation system: A coordinate-based meta-analysis of BOLD fMRI experiments examining neural correlates of subjective value. NeuroImage, 76, 412-427. doi:10.1016/j.neuroimage.2013.02.063

Bechara, A., Damasio, A. R., Damasio, H., \& Anderson, S. W. (1994). Insensitivity to future consequences following damage to human prefrontal cortex. Cognition, 50(1-3), 7-15. doi:10.1016/0010-0277(94)90018-3

Bechara, A., Damasio, H., Tranel, D., \& Damasio, A. R. (1997). Deciding advantageously before knowing the advantageous strategy. Science, 275(5304), 1293-1295. doi:10.1126/ science. 275.5304 .1293

Bechara, A., \& Martin, E. M. (2004). Impaired decision making related to working memory deficits in individuals with substance addictions. Neuropsychology, 18(1), 152-162. doi:10. 1037/0894-4105.18.1.152

Beck, A. T., Steer, R. A., Ball, R., \& Ranieri, W. (1996). Comparison of Beck Depression Inventories-IA and -II in psychiatric outpatients. Journal of Personality Assessment, 67(3), 588-597. doi:10.1207/s15327752jpa6703_13

Buelow, M. T., \& Suhr, J. A. (2009). Construct validity of the Iowa Gambling Task. Neuropsychology Review, 19(1), 102-114. doi:10.1007/s11065-009-9083-4

Carbonell, X., \& Panova, T. (2017). A critical consideration of social networking sites' addiction potential. Addiction Research and Theory, 25(1), 48-57. doi:10.1080/16066359. 2016.1197915

Dancy, C. L., \& Ritter, F. E. (2017). IGT-Open: An open-source, computerized version of the Iowa Gambling Task. Behavior Research Methods, 49(3), 972-978. doi:10.3758/s13428-0160759-4

Delaney, D., Stein, L. A. R., \& Gruber, R. (2017). Facebook addiction and impulsive decision-making. Addiction Research \& Theory, 26(6), 478-486. doi:10.1080/16066359.2017.1406482

Facebook. (2018). Statistics of Facebook. Retrieved from http:// newsroom.fb.com/company-info/

Fernie, G., \& Tunney, R. J. (2006). Some decks are better than others: The effect of reinforcer type and task instructions on learning in the Iowa Gambling Task. Brain and Cognition, 60(1), 94-102. doi:10.1016/j.bandc.2005.09.011

Griffiths, M. D., Kuss, D. J., \& Demetrovics, Z. (2014). Social networking addiction: An overview of preliminary findings. In K. P. Rosenberg \& L. C. Feder (Eds.), Behavioral addictions: Criteria, evidence, and treatment (pp. 119-141). New York, NY: Elsevier Inc.

He, Q., Turel, O., Brevers, D., \& Bechara, A. (2017). Excess social media use in normal populations is associated with amygdalastriatal but not with prefrontal morphology. Psychiatry Research: Neuroimaging, 269, 31-35. doi:10.1016/j.pscychresns. 2017.09.003

He, Q., Xue, G., Chen, C., Lu, Z., Dong, Q., Lei, X., Ding, N., Li, J., Li, H., Chen, C., Li, J., Moyzis, R. K., \& Bechara, A. (2010). Serotonin transporter gene-linked polymorphic region (5-HTTLPR) influences decision making under ambiguity and risk in a large Chinese sample. Neuropharmacology, 59(6), 518-526. doi:10.1016/j.neuropharm.2010.07.008

Karaiskos, D., Tzavellas, E., Balta, G., \& Paparrigopoulos, T. (2010). P02-232 - Social network addiction: A new clinical disorder? European Psychiatry, 25(Suppl. 1), 855. doi:10.1016/ S0924-9338(10)70846-4

Meshi, D., Morawetz, C., \& Heekeren, H. R. (2013). Nucleus accumbens response to gains in reputation for the self relative to gains for others predicts social media use. Frontiers in Human Neuroscience, 7, 439. doi:10.3389/fnhum.2013. 00439

Meshi, D., Tamir, D. I., \& Heekeren, H. R. (2015). The emerging neuroscience of social media. Trends in Cognitive Sciences, 19(12), 771-782. doi:10.1016/j.tics.2015.09.004

Must, A., Szabó, Z., Bódi, N., Szász, A., Janka, Z., \& Kéri, S. (2006). Sensitivity to reward and punishment and the prefrontal cortex in major depression. Journal of Affective Disorders, 90(2-3), 209-215. doi:10.1016/j.jad.2005.12.005

Schmitz, F., Kunina-Habenicht, O., Hildebrandt, A., Oberauer, K., \& Wilhelm, O. (2018). Psychometrics of the Iowa and Berlin gambling tasks: Unresolved issues with reliability and validity for risk taking. Assessment. Advance online publication. 1-14. doi:10.1177/1073191117750470

Statista. (2018). Number of global social network users 2010 2021. Retrieved from https://www.statista.com/statistics/ 278414/number-of-worldwide-social-network-users/

Stevens, L., Verdejo-García, A., Goudriaan, A. E., Roeyers, H., Dom, G., \& Vanderplasschen, W. (2014). Impulsivity as a vulnerability factor for poor addiction treatment outcomes: A review of neurocognitive findings among individuals with substance use disorders. Journal of Substance Abuse Treatment, 47(1), 58-72. doi:10.1016/j.jsat.2014.01.008

Stewart, J. B. (2016). Facebook has 50 minutes of your time each day. It wants more. Retrieved from https://www.nytimes.com/ 2016/05/06/business/facebook-bends-the-rules-of-audienceengagement-to-its-advantage.html

Suckling, J., \& Nestor, L. J. (2017). The neurobiology of addiction: the perspective from magnetic resonance imaging present and future. Addiction, 112(2), 360-369. doi:10.1111/ add. 13474 